\title{
Adaptive Adjustment of Compressed Measurements for Wideband Spectrum Sensing
}

\author{
Yulong Gao, Wei Zhang, Yongkui Ma
}

Harbin Institute of Technology Communication Research Center, Harbin, China, 150001

[e-mail: ylgao@hit.edu.cn; zhangweiand369@163.com; yk_ma@hit.edu.cn]

Received July 21, 2015; revised September 9, 2015; revised October 10, 2015; accepted October 18, 2015; published January 31, 2016

\begin{abstract}
Compressed sensing (CS) possesses the potential benefits for spectrum sensing of wideband signal in cognitive radio. The sparsity of signal in frequency domain denotes the number of occupied channels for spectrum sensing. This paper presents a scheme of adaptively adjusting the number of compressed measurements to reduce the unnecessary computational complexity when priori information about the sparsity of signal cannot be acquired. Firstly, a method of sparsity estimation is introduced because the sparsity of signal is not available in some cognitive radio environments, and the relationship between the amount of used data and estimation accuracy is discussed. Then the SNR of the compressed signal is derived in the closed form. Based on the SNR of the compressed signal and estimated sparsity, an adaptive algorithm of adjusting the number of compressed measurements is proposed. Finally, some simulations are performed, and the results illustrate that the simulations agree with theoretical analysis, which prove the effectiveness of the proposed adaptive adjusting of compressed measurements.
\end{abstract}

Keywords: Wideband Spectrum Sensing; Compressed Sensing; Adaptive Compressed Measurement; SNR of the Compressed Signal. 


\section{Introduction}

$\mathbf{W}_{\text {ith the rapid development of wireless communication over the last decades, the spectrum }}$ resources become increasingly scarce. Furthermore, some of the assigned spectrums are sometimes unoccupied by PUs (primary users), which results in the waste of spectrum resources. In order to exploit the spectrum more efficiently, cognitive radio (CR) has been proposed. Without bad interference to any PU, the SUs (secondary users) could detect the spectrum holes and then access to them [1]-[2]. It is widely recognized that spectrum sensing is a key technology of cognitive radio.

At the beginning, the focus of spectrum sensing was placed on the dectection of narrowband signal. Many typical approaches have been suggested, such as energy-based detection, matched-filter detection and cyclic spectral detection [3]. However, with the increasing requirement of future high-speed cognitive data, detection of wideband signal becomes a hot topic.

Up to now, many detection algorithms of wideband signal have been reported [4]-[9]. The first scheme is a direct generalization of conventional narrowband methods. Wideband signal is divided into many narrowband signals, and these narrowband signals are detected one by one. Because these methods require much operation time, the algorithms of simultaneously and parallelly processing the wideband signal were proposed [4]-[5]. Typical algorithms mainly consist of filter band spectrum sensing [4], multi-resolution spectrum sensing [5]. For the filter band spectrum sensing, each channel is equipped with band-pass filter, which results in too high hardware complexity and cost. As for multi-resolution spectrum sensing, the wideband signal is detected in two stages: a sparse stage and a precise stage, where the wavelet transformation is always exploited [6]. In the case of multi-resolution spectrum sensing, much more time and computational complexity are required when the bandwidth of signal increases. In addition, to cope with the multi-path fading and shadow of wireless channel, collaborative spectrum sensing methods of wideband signal have been addressed [7]-[8]. Generally, two cases are considered. The first case is that each SU detects the entire channel, where Markov process is an important mathmatical tool. The second case is that only one channel is detected by one SU, and many SU are exploited to detect all of channels. It is widely accepted that wideband ADC is required for the forementioned approaches. However, according to Nyquist sampling theory, it is necessary to sample the signal at least twice faster than its bandwidth, which is a challenging task for ADC hardware and cost [9]-[10]. Moreover, a large amount of sampled signal results in high computational complicity.

Fortunately, the compressed sensing (CS) makes it possible to sample the signal at a slower rate than Nyquist sampling rate [11]. According to CS theory, the sparsity of signal denotes the number of non-zero values in some basis [12], and a sparse signal can be accomplished through the linear random projections. Moreover, the signal can be reconstructed through the compressed measurements with high probability [13]. Based on these advantages of CS, a few CS-based spectrum sensing algorithms of wideband signal have been proposed in [9],[14]-[16]. Z.Tian and G.B.Giannakis introduced the compressed sensing to spectrum sensing of wideband signal firstly [14], then a cross-correlation-based method was addressed with cyclic spectral density [15]. In [9], wideband spectrum sensing was modeled as a well-known structured covariance estimation problem because the compressed measurements are a linear combination of the original signals. In order to reduce the computational complexity, a novel decomposition compressed spectrum sensing scheme was presented [10]. 
In addition, some cooperative compressed spectrum sensing algorithms have been addressed by terms of advantages of cooperative spectrum sensing and compressed sensing [16].

For previous CS-based spectrum sensing of wideband signal, signal reconstruction is the first and key step, and then reconstructed signal is processed with different mathematical tools to derive test statistic [9],[14]-[16], which also poses high computational complexity. At present, the adopted reconstruction methods are mainly divided into two categories: convex relaxation algorithms and iterative greedy algorithms [17-20]. For convex relaxation algorithms, sparseness constraint is changed from $L_{0}$-norm to $L_{1}$-norm. They consist of BP (Basis Pursuit), BPDN (Basis Pursuit Denoise), $S L_{0}$ (Smoothed $L_{0}$ ), which can be realized by exploiting an interior-point method for noise-free case and gradient projection for noise case [17]. Computational complexity of BP is $O\left(N^{3}\right)$, which is very high for practical application. To cope with this challenge, some iterative greedy algorithms have been proposed [18-20]. Reconstuction error is permitted under the constraint of minimum $L_{0}$-norm for iterative greedy algorithms. Typical algorithms include MP (Matching Pursuit), OMP (Orthogonal Matching Pursuit) and the modified methods of OMP. Matching pursuit is the most basic method, but its convergence is slow. Then OMP was presented for solving these existing problems of MP [18], and its computational complexit is $O(K M N)$, which is far less than that of BP. OMP is a non-linear adaptive algorithm based on MP, and the selected itom is orthogonalized to reach faster convergence rate and higher reconstruction accuracy. Subsequently, some modified algorithms of OMP were introduced, such as ROMP (Regularized Orthogonal Matching Pursuit), CoSaMP (Compressive Sampling Matching Pursuit) and StOMP (Stagewise OMP) [19-20]. Each of these algorithms has its own merits and weaknesses, for example, priori information about sparsity of signal and noise is required by OMP, and threshold decision is tightly correlated to measurement matrix for StOMP, which seriously limits its applications. Considering recovery accuracy, computational complexity and feasibility comprehensively, OMP algorithm is always exploited for many applications by most of literatures.

We can see that high computational complexity is caused by reconstruction algorithm. It has been proved that the accuracy of reconstruction and computational complexity are decided by the number of compressed measurements for reconstruction algorithm [14]-[16]. For iterative greedy algorithm, the number of compressed measurements is a key factor of impacting the computational complexity. Too many compressed measurements lead to very high computational complexity, and too little compressed measurements have a severe impact on the performance of reconstruction. Therefore, a few adaptive reconstruction methods have been introduced. An adaptive sensing and group testing algorithm for sparse signal reconstruction was proposed in [21]-[24]. In terms of sparse target scene, an adaptive method was addressed in [25], where the measurement matrix can be updated by the target scene information fed back by the reconstruction algorithm. In [26], total coefficients power was defined as a new metric to guide the node selection to build a sparse additional projection vector. In addition, the necessary number of compressed measurements was proposed to reconstruct a sparse signal in a required probability [27]. However, the practical signal is always corrupted by noise, and the real sparse signals scarcely exist. As a result, the methods in [21]-[27] can be only applied in noise-free scenario, which is unsuited for spectrum sensing. Most importantly, the SNR of compressed signal has a direct impact on the performance of spectrum sensing, which is dominated by the number of compressed measurements. Therefore, we will explore the relationship between the SNR of the compressed signal and the number of compressed measurements. What's more, other factors should also be considered to obtain appropriate number of compressed measurements and closed-form SNR of compressed signal, such as the non-orthogonality of measurement matrix, the sparsity of signal, coherence of 
vectors of measurement matrix. To sum up, the proposed spectrum sensing can be described as follows. Firstly, the $S N R$ of the compressed signal is calculated according to the requirements of spectrum sensing performance, estimated sparsity of signal and SNR of the non-compressed signal. And then the appropriate number of compressed measurements is acquired by the SNR of the compressed signal. Finally, the frequency-domain signal is reconstructed by virtue of compressed measurements to finish spectrum sensing. From the proposed algorithm, we can see that the number of compressed measurements varies adaptively with $S N R$ of the compressed signal, which can decrease the computational complexity of spectrum sensing.

For current CS-based spectrum sensing of wideband signal, another factor of high computaional complexity is calculating test statistic and obtaining time-domain signal from sparse frequency-domain signal. Therefore, we exploit directly the reconstructed frquency-domain signal to finish spectrum sensing, namely the frequency-domain non-zero values of reconstructed signal denote the existence of PUs. Consequently, this scheme removes the two before-mentioned mathmatical operations, which can further decrease the computational complexity.

It should also be noted that the previous methods are supposed to know the sparsity of signal. However, random appearance of PUs leads to time-varying sparsity for cognitive radio environments. Therefore, sparsity estimation must be firstly performed for spectrum sensing. Many works on sparsity estimation have been reported in past few years. A novel scheme of sparsity estimation was presented by means of the divide and conquers method in [28]. In [29], an eigenvalue-based compressive $\mathrm{SOE}$ technique was proposed in terms of asymptotic random matrix theory. In addition, A SNR-based sparsity estimation method was introduced to detect the sparsity level of the channel in [30]. Sparsity estimation for different kinds of random matrix has been discussed in [31]-[32]. For these methods, the sparsity estimation is quite accurate if enough data are exploited, which poses the high computational complexity and long sampling time. As a consequence, it is not comprehensive to evaluate the estimation methods only by estimation accuracy.

Our main contributions are: 1) a method of sparsity estimation is proposed, whose performance is theoretically analyzed by the amount of the used data and estimation accuracy. 2) The closed-form expression of SNR of the compressed signal is obtained, and it can be concluded that three factors (SNR of the non-compressed signal, the sparsity of signal and number of compressed measurements) have an impact on SNR of the compressed signal. 3) We introduce the approach to adaptively adjust the compressed measurements for the two different cases.

The rest of the paper is organized as follows. In section 2, we introduce the system model for wideband spectrum sensing. In section 3 , we propose a method of sparsity estimation and analyze its performance theoretically. In addition, the relationship between the amount of the used data and estimation accuracy is obtained. In section 4, the SNR of the compressed signal is derived in the closed form, and the specific process of the proposed spectrum sensing is described. In addition, the method of adaptively adjusting the number of compressed measurements is proposed for the two typical sensing scenarios. In section 5, some simulations are performed to validate theoretical analysis. We also compare the proposed method with the traditional schemes for the various performance metrics. Finally, we conclude the paper in Section 6. 


\section{System Model of Wideband Spectrum Sensing}

In this section, a system model for wideband spectrum sensing is addressed. Suppose that discrete Fourier transformation of wideband signal $\boldsymbol{r}$ is $\boldsymbol{x}=\boldsymbol{F}(\boldsymbol{r})$, which is a $N \times 1$ vector in frequency domain, and only $K(K<<N)$ entries of $\boldsymbol{x}$ are non-zero. Namely, the signal $\boldsymbol{r}$ is sparse in frequency domain. According to CS theory, such a signal can be accomplished through the linear random projections

$$
\boldsymbol{y}=\boldsymbol{\Theta x}
$$

Here, $\Theta$ is a $M$ by $N$ random matrix with the condition $M<<N$, and $K$ denotes the sparsity of signal in Fourier basis[12], which is the number of occuped bands in our proposed spectrum sneing method. Through linear projections, $M$ measurements rather than $N$ sampling signal is obtained. It should be noted that random matrix $\Theta$ is required to satisfy restricted isometry property (RIP). Namely, a matrix $\boldsymbol{\Theta}_{M \times N}$ satisfies the RIP of order $K$ if there exists a constant $\delta_{K}, 0<\delta_{K}<1$, for all $\|\boldsymbol{x}\|_{0}<K$, we have

$$
\left(1-\delta_{K}\right)\|\boldsymbol{x}\|_{2}^{2}<\|\boldsymbol{\Theta} \boldsymbol{x}\|_{2}^{2}<\left(1+\delta_{K}\right)\|\boldsymbol{x}\|_{2}^{2}
$$

What's more, we assume that the maximum number of PUs is $N$ in frequency band $(0 \sim W \mathrm{~Hz})$, and each of PUs occupies a channel of $W / N H z$. However, only a small fraction of $N$ PUs exploit the channel at a given time and location. We assume that only $K(K<<N)$ channels are adopted by active PUs, and the remaining $N-K$ channels are idle. Clearly, the wideband signal $\boldsymbol{x}$ of PUs is $K$-sparse in the frequency domain. Then the idle $N-K$ channels provide spectrum opportunity for the SUs. Fig. 1 shows spectrum occupancy of PUs.

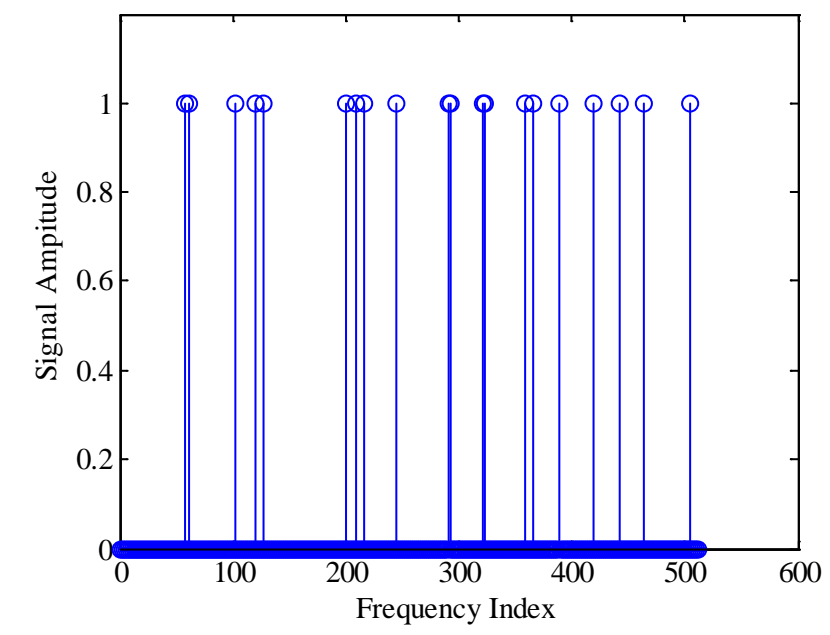

Fig. 1. An illustration of wideband spectrum occupancy by PUs, for the cases of $N=512$ and $K=20$, assuming that all the occupied channels have the unit amplitude.

Considering the impact of wireless channel, the compressed measurements of SU's are expressed in the form

$$
\boldsymbol{y}=\boldsymbol{\Phi} \mathbf{F}^{-1} \boldsymbol{H} \boldsymbol{x}+\boldsymbol{n}
$$

where y and $\mathrm{x}$ are a $M$-dimension complex vector and a $N$-dimension complex vector, denoting the compressed measurements and the Fourier coefficients of PUs' signal respectively. And $M$ is the number of compressed measurements. When $K(K<<N)$ channels 
are occupied by active PUs, only $K$ entries in $\boldsymbol{x}$ are non-zero. The matrix $\boldsymbol{\Phi}$ represents a $M$ by $N$ Bernoulli random matrix, here we exploit the AIC model presented in [14]. $\mathbf{F}^{-1}$ is an inverse discrete Fourier transformation matrix. $\boldsymbol{H}=\operatorname{diag}(h(1), h(2), \ldots h(N))$ is the channel matrix between SU and PUs, where $h(i)(i=1,2, \ldots, N)$ denotes the channel gain for the $i^{\text {th }}$ channel. As we mentioned above, the $i^{\text {th }}$ channel occupies the spectrum of $\left[(i-1) \frac{W}{N}, i \frac{W}{N}\right] H z$ in frequency domain. In addition, $\boldsymbol{n}$ represents a Gaussian noise vector. By simplifying equation $\boldsymbol{\Theta}=\boldsymbol{\Phi} \mathbf{F}^{-1} \boldsymbol{H}$, we can get $\boldsymbol{y}=\boldsymbol{\Theta} \boldsymbol{x}+\boldsymbol{n}$. In CR environments, the purpose of spectrum sensing is to detect the locations of $K$ channels occupied by PUs, and then SUs can take advantage of other idle $N-K$ channels. Here, the compressed measurements vector $\boldsymbol{y}$ and the matrix $\boldsymbol{\Theta}$ are available information for spectrum sensing. In summary, the problem of spectrum sensing is translated to the CS reconstruction under the constraint of more conditions, which can be expressed as

$$
\hat{\boldsymbol{x}}=\arg \min \|\boldsymbol{x}\|_{0}, \quad \text { s.t. } \quad \boldsymbol{y}=\boldsymbol{\Theta} \boldsymbol{x} \text { and some additional conditions }
$$

The additional conditions are as follows, the sparsity of signal $K$ is unknown and the number of compressed measurements is varied.

According to the forementioned model, we carry out spectrum sensing by reconstructing the sparse signal in frequency domain. In other words, reconstructed frequency-domain signal represents the result of spectrum sensing. What is more, no other further operation is required for spectrum sensing.

\section{Method of Sparsity Estimation}

In this section, we introduce a method of sparsity estimation and analyze the performance theoretically.

Lemma 1: If $\left\|\boldsymbol{x}_{1}\right\|_{0}=K,\left\|\boldsymbol{x}_{2}\right\|_{0}=K+1,\left\|\boldsymbol{\Theta} \boldsymbol{x}_{1}\right\|_{2}^{2}<\left\|\boldsymbol{\Theta} \boldsymbol{x}_{2}\right\|_{2}^{2}$ holds when a matrix $\boldsymbol{\Theta}$ satisfies the RIP of order $K$ with isometry constant $\delta<\frac{1}{2 K+1}$ and the received signals possess the similar power and experience a slow fading.

Proof: Considering that the received signals possess similar power, namely the non-zero entries of $x$ approximate to each other and are assumed as $a$. If the sparsity of $\boldsymbol{x}_{1}$ is $K$, then $\left\|x_{1}\right\|_{2}^{2} \approx K a^{2}$. Substituting this result into the expression of RIP, we can get

$$
(1-\delta) K a^{2} \leq\left\|\boldsymbol{\Theta} x_{1}\right\|_{2}^{2} \leq(1+\delta) K a^{2}
$$

Similarly, when the signal experiences a slow fading, the non-zero entries of $\boldsymbol{x}_{1}$ and $\boldsymbol{x}_{2}$ in the same indices can be viewed as an unchanged value during coherence time. Thus, for $\boldsymbol{x}_{2}$ we have

$$
(1-\delta)(K+1) a^{2} \leq\left\|\boldsymbol{\Theta} x_{2}\right\|_{2}^{2} \leq(1+\delta)(K+1) a^{2}
$$

If $\left\|\boldsymbol{\Theta} \boldsymbol{x}_{1}\right\|_{2}^{2}<\left\|\boldsymbol{\Theta} \boldsymbol{x}_{2}\right\|_{2}^{2}$, the expression $(1+\delta) K a^{2}<(1-\delta)(K+1) a^{2}$ must be satisfied. Through simplifying the expression, $\delta<\frac{1}{2 K+1}$ can be obtained. So, it is proved that if $\left\|\boldsymbol{x}_{1}\right\|_{0}=K$ and $\left\|\boldsymbol{x}_{2}\right\|_{0}=K+1,\left\|\boldsymbol{\Theta} \boldsymbol{x}_{1}\right\|_{2}^{2}<\left\|\boldsymbol{\Theta} \boldsymbol{x}_{2}\right\|_{2}^{2}$ holds. It means that higher sparsity of signal $\boldsymbol{x}$ poses larger 
value of $\|\boldsymbol{\Theta x}\|_{2}^{2}$, which provides premise and foundation to estimate the sparsity of signal.

Suppose that a matrix $\Theta$ satisfies the RIP of order $K$ with isometry constant $\delta$, we have $(1-\delta) \leq \frac{\|\boldsymbol{\Theta} \boldsymbol{x}\|_{2}^{2}}{\|\boldsymbol{x}\|_{2}^{2}} \leq(1+\delta)$. Because of very small isometry constant $\delta, \frac{\|\boldsymbol{\Theta} \boldsymbol{x}\|_{2}^{2}}{\|\boldsymbol{x}\|_{2}^{2}}$ approximates to 1, e.g., $\|\boldsymbol{\Theta} \boldsymbol{x}\|_{2}^{2} \approx\|\boldsymbol{x}\|_{2}^{2}$. Therefore, the estimated value of $\|\boldsymbol{x}\|_{2}^{2}$ can be obtained in terms of $\|\boldsymbol{\Theta} \boldsymbol{x}\|_{2}^{2}$. If the non-zero entries of vector $\boldsymbol{x}$ approximate to $a$, we have $\|\mathbf{x}\|_{2}^{2} \approx a^{2}\|\mathbf{x}\|_{0}=a^{2} K^{\prime}$. Thus, the estimated sparsity of $x$ can be acquired from the following expression

$$
K^{\prime}=\frac{\|x\|_{2}^{2}}{a^{2}} \approx \frac{\|\boldsymbol{\Theta} x\|_{2}^{2}}{a^{2}}
$$

It is clear that $\|\boldsymbol{x}\|_{2}^{2}$ and $\|\boldsymbol{\Theta} x\|_{2}^{2}$ vary with $\delta$ proportionally. Therefore, the estimated sparsity of signal is more accurate when $\delta$ becomes smaller.

According to previous analysis, we can see that equation (6) holds when two conditions are satisfied. The first condition is that the non-zero entries of vector $\boldsymbol{x}$ in the same time approximate to each other, which requires similar power for the received signals in the different frequency band. Therefore, it is relatively strict for some practical applications. The second condition is that the non-zero entries of vectors $\boldsymbol{x}_{1}$ and $\boldsymbol{x}_{2}$ in the same indices approximate to each other, where $\boldsymbol{x}_{1}$ and $\boldsymbol{x}_{2}$ are vectors in different time during coherence time, but when time difference of $\boldsymbol{x}_{1}$ and $\boldsymbol{x}_{2}$ exceeds the coherence time, they will have different value. This condition can be satisfied when the signals experience a slow fading. If the previous two conditions can not be satisfied for practical circumstances, we can employ other methods introduced in [28]-[32]. As described in section 1, these methods can perform well. Compared with the proposed method, they only have relatively high computational complexity. In addition, the estimation methods of sparsity do not affect the performance of wideband spectrum sensing if only we can get the correct sparsity of signal.

\section{Adaptive adjustment of Compressed Measurements}

In this section, we analyze the $S N R$ of the compressed signal, and conclude that three parts ( $S N R$ of the non-compressed signal, the sparsity of signal and number of compressed measurements) will influence the SNR of the compressed signal. Based on the SNR of the compressed signal, we propose a method of adjusting the number of compressed measurements adaptively.

\subsection{Derivation of SNR of the Compressed Signal}

Consider that $\boldsymbol{x}$ is a $N \times 1$ signal vector and only $K(K<<N)$ entries of $x$ are non-zero. According to CS theory, such a signal can be compressed by the linear random projections $\boldsymbol{y}=\boldsymbol{\Theta} \boldsymbol{x}$, where $\boldsymbol{\Theta}$ denotes a $M$ by $N(M<<N)$ random matrix. Here, let $\Lambda=\operatorname{supp}(\boldsymbol{x})$ denote the set of indices for the non-zero coordinates of the signal vector $x$. Using the set $\Lambda$, the measurement vector $\boldsymbol{y}$ is expressed as

$$
\boldsymbol{y}=\sum_{i \in \Lambda} \boldsymbol{\Theta}_{i} x_{i}
$$

Where $\boldsymbol{\Theta}_{i}$ denotes the $i^{\text {th }}$ column of the matrix $\boldsymbol{\Theta}$, and $x_{i}$ is the entry of the signal $\boldsymbol{x}$ with index $i$. This equation implies that measurement vector $\boldsymbol{y}$ is the linear combination of the 
columns of $\boldsymbol{\Theta}$, and the coefficients are $x_{i}(i \in \Lambda)$. In other words, the non-zero entries of $x_{i}(i \in \Lambda)$ are projected to columns of $\boldsymbol{\Theta}_{i}$, and then their sum is calculated.

In order to clearly describe the noise and interference brought by compressed sensing, we take a simple case for example. Let $\boldsymbol{x}=\left[x_{1}, x_{2}, x_{3}\right]^{T}$. The measurement matrix $\boldsymbol{\Theta}$ is assumed as

$$
\boldsymbol{\Theta}=\left[\boldsymbol{\Theta}_{1}, \boldsymbol{\Theta}_{2}, \boldsymbol{\Theta}_{3}\right]=\left[\begin{array}{lll}
1 & 0 & 0 \\
0 & 1 & 0 \\
0 & 0 & 1
\end{array}\right]
$$

Thus, the measurement matrix indicates that the signal $x$ is not compressed. Fig. 2 illustrates three unit vectors $\boldsymbol{\Theta}_{1}, \boldsymbol{\Theta}_{2}$ and $\boldsymbol{\Theta}_{3}$ in the three-dimension coordinate system, which denote $\mathrm{X}$-axis basis vector, $\mathrm{Y}$-axis basis vector and Z-axis basis vector respectively. It can be seen from equation (7) that the measurement vector $y$ denotes a point $\left\{x_{1}, x_{2}, x_{3}\right\}$ in three-dimension coordinate system when the entries of $\boldsymbol{x}$ are projected.

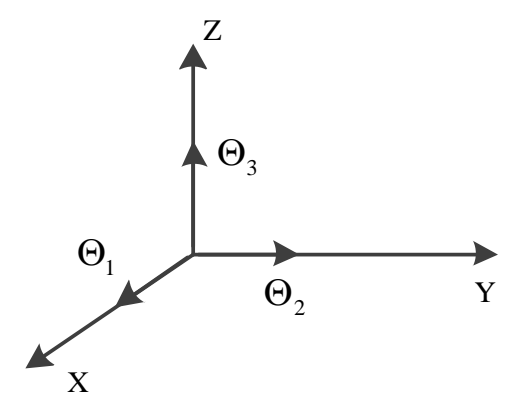

Fig. 2. Three basis vectors in three-dimension coordinate system.

If there exists noise, the actual signal can be expressed as $\boldsymbol{r}=\boldsymbol{x}+\boldsymbol{n}=\left[x_{1}+n_{1}, x_{2}+n_{2}, x_{3}+n_{3}\right]$. Just like $x_{i}$, the noise $n_{i}$ is also projected to $\mathrm{X}$-axis, Y-axis and Z-axis respectively. As we see in Fig. 2, $n_{j}$ have no projection on $\boldsymbol{\Theta}_{i}(i \neq j)$ because three basis vectors $\boldsymbol{\Theta}_{1}, \boldsymbol{\Theta}_{2}, \boldsymbol{\Theta}_{3}$ are mutually orthogonal. When $x_{i}$ is projected to $\boldsymbol{\Theta}_{i}$, the $S N R_{i}$ of each dimension is written as

$$
S N R_{i}=\frac{\left\|x_{i}\right\|_{2}^{2}}{\left\|n_{i}\right\|_{2}^{2}}, i=1,2,3
$$

If compressed sensing is applied, here we take $M=2, N=3$ for example, $\boldsymbol{\Theta}=\left[\boldsymbol{\Theta}_{1}, \boldsymbol{\Theta}_{2}, \boldsymbol{\Theta}_{3}\right]$ is a 2 by 3 matrix. Three vectors in two-dimension coordinate system are shown in Fig. 3.

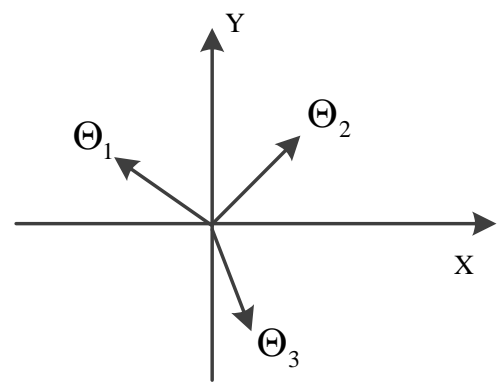

Fig. 3. Three basis vectors in two-dimension coordinate system 
The measurement vector y can be expressed as

$$
\begin{aligned}
\boldsymbol{y} & =\boldsymbol{\Theta}_{1} r_{1}+\boldsymbol{\Theta}_{2} r_{2}+\boldsymbol{\Theta}_{3} r_{3} \\
& =\boldsymbol{\Theta}_{1}\left(x_{1}+n_{1}\right)+\boldsymbol{\Theta}_{2}\left(x_{2}+n_{2}\right)+\boldsymbol{\Theta}_{3}\left(x_{3}+n_{3}\right)
\end{aligned}
$$

It is clear that these three vectors $\boldsymbol{\Theta}_{1}, \boldsymbol{\Theta}_{2}, \boldsymbol{\Theta}_{3}$ cannot be mutually orthogonal. Therefore, $n_{i}$ is projected to $\boldsymbol{\Theta}_{j}(i \neq j)$, and the projection coefficient $c_{i j}=\operatorname{dot}\left(\boldsymbol{\Theta}_{i}, \boldsymbol{\Theta}_{j}\right)$, where $\operatorname{dot}() \operatorname{denotes}$ inner product of vectors. Apart from $n_{i}, x_{i}$ is also projected to $\boldsymbol{\Theta}_{j}(i \neq j)$, and the projection coefficient is $c_{i j}=\operatorname{dot}\left(\boldsymbol{\Theta}_{i}, \boldsymbol{\Theta}_{j}\right)$. It can be seen that the noise brought by compressed sensing consists of the non-compressed noise $n_{i}$, the projection of other noise $n_{j} c_{i j}$ and the projection of non-zero signal $x_{k} c_{k i}$.

From the previous analysis, the SNR of the compressed signal is denoted as

$$
S N R^{\prime}=E\left[S N R_{i}\right]=E\left[\frac{\left|x_{i}\right|^{2}}{\left|x_{i}+\sum_{j=1, i \neq j}^{N} n_{j} c_{i j}+n_{i}+\sum_{k=1, k \neq i}^{K} x_{k} c_{k i}\right|^{2}-\left|x_{i}\right|^{2}}\right]
$$

Where $S N R^{\prime}$ denotes $S N R$ of the compressed signal, and $E($ ) refers to the mathematical expectation.

In order to simplify equation (11), we assume that noise vector $\boldsymbol{n}$ consists of independently and identically distributed (i.i.d.) Gaussian components with mean zero and variance $\sigma^{2}$. The signal vector $x$ consists of i.i.d. Bernoulli components, which satisfy $P\left[x_{i}=1\right]=\lambda$ and $P\left[x_{i}=0\right]=1-\lambda$, where $\lambda$ refers to the parameter of Bernoulli, and we have $K=N \lambda$. Let $\boldsymbol{C}=\boldsymbol{\Theta}^{T} \boldsymbol{\Theta}$ denotes a $M$ by $N$ matrix with i.i.d. non-diagonal components, and we have $c_{i j}=\operatorname{dot}\left(\boldsymbol{\Theta}_{i}, \boldsymbol{\Theta}\right)_{j}(i \neq j)$. The mathematical expectation of $c_{i j}(i \neq j)$ is 0 , and $E\left(c^{2}\right)=E\left(c_{i j}^{2}\right)$ holds, which will be discussed in the section 5 . Based on the previous discussion, the following expression can be obtained

$$
\begin{aligned}
S N R^{\prime} & =E\left[\frac{\left|x_{i}\right|^{2}}{\left|x_{i}+\sum_{j=1, i \neq j}^{N} n_{j} c_{i j}+n_{i}+\sum_{k=1, k \neq i}^{K} x_{k} c_{k i}\right|^{2}-\left|x_{i}\right|^{2}}\right] \\
& =\frac{\left(x_{i}\right)^{2}}{\sum_{j=1, i \neq j}^{N} E\left(n_{j} c_{i j}\right)^{2}+E\left(n_{i}\right)^{2}+\sum_{k=1, k \neq i}^{K} E\left(x_{k} c_{k i}\right)^{2}} \\
& =\frac{S N R}{(N-1) E\left(c^{2}\right)+1+(K-1) \times S N R \times E\left(c^{2}\right)}
\end{aligned}
$$

Where $S N R$ denotes the signal noise ratio of the non-compressed signal. According to equation (12), three conclusions can be obtained: (1) $S N R^{\prime}$ is related to the $S N R$ of the non-compressed signal, and it is less than the $S N R$ of the non-compressed signal because the denominator is more than 1. It indicates that compressed sensing decreases $S N R$. (2) SNR' 
varies with mathematical expectation $E\left(c^{2}\right)$ inversely. Meanwhile, $E\left(c^{2}\right)$ is related to the measurement matrix, which will be discussed in section 5. (3) If the sparsity of signal experiences a decline, $S N R^{\prime}$ will increase accordingly.

\subsection{Calculation of SNR of the Compressed Signal}

Consider equation (12), in order to calculate the $S N R$ of the compressed signal ( $S N R^{\prime}$ ), the $S N R$ of the non-compressed signal and $E\left(c^{2}\right)$ are known as the priori knowledge generally. Because many methods of calculating $S N R$ of the non-compressed signal have been reported, and it is not our emphasis, we don't address corresponding algorithm in detail. Below, we provide a method of calculating $E\left(c^{2}\right)$. Because $c_{i j}=\left(\boldsymbol{\Theta}^{T} \boldsymbol{\Theta}\right)_{i j}, i \neq j$ is i.i.d., the probability density function (PDF) $f(c)$ can be denoted by virtue of $f\left(c_{i j}\right), i \neq j$. By the definition of mathematical expectation, $E\left(c^{2}\right)$ is calculated by

$$
E\left(c^{2}\right)=\int c^{2} f(c) d c
$$

Furthermore, we have $c_{i j}=\boldsymbol{\Theta}_{i} \cdot \boldsymbol{\Theta}_{j}=\left\|\boldsymbol{\Theta}_{i}\right\|_{2} \times\left\|\boldsymbol{\Theta}_{j}\right\|_{2} \cos \theta_{i j}$, where $\theta_{i j}$ is the angle between two vectors $\boldsymbol{\Theta}_{i}$ and $\boldsymbol{\Theta}_{j}$. If the columns of the measurement matrix $\boldsymbol{\Theta}$ are normalized, which indicates that $\left\|\boldsymbol{\Theta}_{i}\right\|_{2}=\left\|\boldsymbol{\Theta}_{j}\right\|_{2}=1, \quad c_{i j}=\cos \theta_{i j}$ is obtained. Similarly, $\theta_{i j}, i \neq j$ is i.i.d., so $f\left(\theta_{i j}\right), i \neq j$ is represented by PDF $f(\theta)$. From the previous analysis, the relationship between PDF $f(\theta)$ and $f(c)$ can be written as

$$
f(\theta) \stackrel{c=\cos \theta}{\Rightarrow} f(c)
$$

Let $F_{c}(c)$ denote cumulative distribution function (CDF) of variable $c$, and $F_{\theta}(\theta)$ indicate CDF of variable $\theta$. Then, the expression can be obtained in the form

$$
\begin{aligned}
F_{c}(c) & =P(\cos \theta \leq c) \\
& =P(\theta \geq \arccos c) \\
& =1-P(\theta \leq \arccos c) \\
& =1-F_{\theta}(\arccos c)
\end{aligned}
$$

After calculating the derivative, the $\operatorname{PDF} f(c)$ can be acquired

$$
\begin{aligned}
f(c) & =-\left.f(\theta)\right|_{\theta=\arccos c} \times(\arccos c)^{\prime} \\
& =\left.f(\theta)\right|_{\theta=\arccos c} \times \frac{1}{\sqrt{1-c^{2}}}
\end{aligned}
$$

The PDF of $f(\theta)$ is given in [33]

$$
\begin{aligned}
& f(\theta)=I_{M} \sin ^{n-2} \theta, \quad \theta \in[0, \pi] \\
& I_{M}= \begin{cases}\frac{(M-2) ! !}{2 \times(M-3) ! !}, & M \text { is odd more than } 3 \\
\frac{(M-2) ! !}{\pi \times(M-3) ! !}, & M \text { is even more than } 2\end{cases}
\end{aligned}
$$

Here, the variable $\theta$ denotes the angle between two column vectors of measurement matrix, and $M$ is the length of two vectors. The operation ( )!! refers to double factorial. By terms of 
equation (16) and equation (17), the PDF of $f(c)$ is obtained. What is more, applying the result of $f(c)$ into equation (13), mathematical expectation $E\left(c^{2}\right)$ is computed, which is only the function of $M$. Finally, $S N R^{\prime}$ can be calculated by employing equation (12).

\subsection{Adaptive Method of Adjusting Compressed Measurements}

Now, equation (12) is rewritten as

$$
S N R^{\prime}=\frac{S N R}{(N-1) E\left(c^{2}\right)+1+(K-1) \times S N R \times E\left(c^{2}\right)}
$$

It has been widely recognized that the $S N R$ of the compressed signal ( $S N R^{\prime}$ ) determines spectrum sensing performance. Specifically speaking, spectrum sensing performance is dominated by the SNR of the non-compressed signal, sparsity of signal and $E\left(c^{2}\right)$. According to characters of three factors, there exist two typical sensing scenarios. The first case is the fixed $S N R$ of the compressed signal and the varied sparsity of signal. Therefore, the SNR of the compressed signal ( $S N R^{\prime}$ ) is influenced by the estimated sparsity of signal $K^{\prime}$ and expectation $E\left(c^{2}\right)$ (the number of compressed measurements). When the estimated sparsity is acquired, we can control the SNR of the compressed signal by changing the number of compressed measurements to achieve a certain spectrum sensing performance under the constraint of computational complexity.

Consider another scenario, the sparsity of signal $K$ is fixed, but the SNR of the non-compressed signal fluctuates. From equation (18), the $S N R$ of the compressed signal varies accordingly. Here, the value of $E\left(c^{2}\right)$ can also be adjusted to guarantee certain $S N R^{\prime}$. Similarly, changing of $E\left(c^{2}\right)$ can be achieved by changing the number of compressed measurements.

In summary, the block diagram of spectrum sensing can be schematically illustrated in Fig. 4. Firstly, the received signal is compressed using a matrix of sparsity estimation $\Theta_{1}\left(M_{1} \times N\right)$, and the compressed measurements are acquired $\mathbf{y}_{1}=\boldsymbol{\Theta}_{1} \boldsymbol{x}$. Then the sparsity $K$ is estimated by terms of $\left\|\mathbf{y}_{1}\right\|_{2}^{2}$. Secondly, the row number of measurement matrix of spectrum sensing is calculated according to the estimated $K$ and the required $S N R$ of compressed sensing, then the received signal is compressed by the measurement matrix of spectrum sensing $\boldsymbol{\Theta}_{2}\left(M_{2} \times N\right)$, therefore compressed measurements are computed by $\mathbf{y}_{2}=\boldsymbol{\Theta}_{2} \boldsymbol{x}$. Lastly, let $\boldsymbol{\Theta}=\left[\boldsymbol{\Theta}_{1}, \boldsymbol{\Theta}_{2}\right]^{T}$ and $\mathbf{y}=\left[\mathbf{y}_{1}, \mathbf{y}_{2}\right]^{T}$, where $\boldsymbol{\Theta}$ and y are an $M$ by $N$ matrix and an $M$-dimension vector respectively, i.e., $M=M_{1}+M_{2}$. Then $\boldsymbol{\Theta}$ and y are exploited to reconstruct frequency-domain signal, and the results of spectrum sensing can be obtained according to reconstructed frequency-domain signal. 


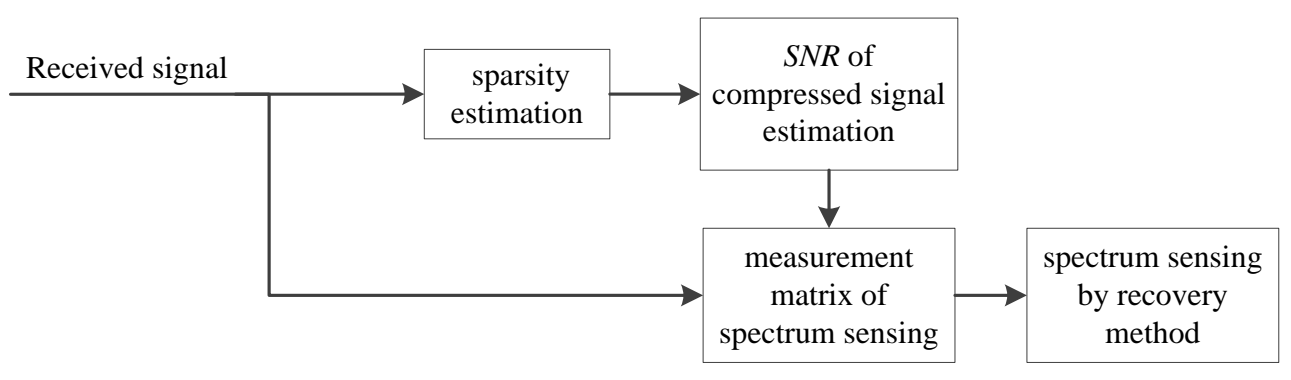

Fig. 4. the process of spectrum sensing using sparsity and SNR estimation

Now, we analyze the computational complexity of proposed algorithm, which consists of sparsity estimation, calculating the SNR of compressed signal and reconstructing frequency-domain signal. It is supposed that the real signal is adopted. Sparsity estiamtion is finised by calculating $L_{2}$-norm, which requires $M_{1}$ multiplications and $M_{1}-1$ additions. By analyzing equation (18), the computational complexity of calculating the SNR of compressed signal only is negligible. As mentioned above, OMP is the reconstrution algorithm used in this paper, and its computational complexit is $O(K M N)$. Based on previous analysis, the reconstruction algorithm need maximum computational complexity in the proposed method. $N$ and $K$ are same for the conventional schemes and the proposed method. However, we can adjust $M$ to reach a minimum value for the required spectrum sensing performance, which is the main cause of alleviating computational complexity. Moreover, as addressed in introduction, eliminating calculations of test statistic and conversion from time-domain signal from sparse frequency-domain signal will also partially reduce the computational sources comparing with previous algorithms.

\section{Numerical Results}

In this section, some simulations are carried out to verify theoretical analysis. In addition, the various performance metrics of the proposed method are compared with that of the traditional methods. Because OMP algorithm is widely exploited and it is a base of other recovery methods, OMP algorithm is selected as reconstruction algorithm in simulations. The following parameters remain fixed throughout all simulations: the length of signal $N=512$ and the times of Monte Carlo is 5000. The other parameters are separately specified for each experiment.

\subsection{Simulation Results for Sparsity Estimation Method}

To evaluate the performance of the proposed method of sparsity estimation, the normalized error $(N E)$ is defined as

$$
N E=D\left[\frac{K-K^{\prime}}{K}\right]
$$

Where $D[], K$ and $K^{\prime}$ denote variance function, the actual sparsity of signal and the estimated sparsity with the proposed method separatively. Normalized error ( $N E$ ) versus the sparsity of signal is presented in Fig. 5. The simulation parameters are as follows. $M=10,40$ 
and 50, respectively. The SNR of non-compressed signal is 30dB. $K$ is from 10 to 90 and the corresponding step length is 10 . The three lines represent the different performances of three compressed measurements separatively. As illustrated in Fig. 5, NE is lower when more compressed measurements are exploited. When the number of compressed measurements is not changed, normalized error can remain stable. According to compressed sensing, more compressed measurements can capture and extract more information about the sparsity of signal from the same signal $\boldsymbol{x}$, which means that more equations can be acquired mathematically. As a consequance, the sparsity estimation error varies with the number of compressed measurements when other parameters are retained.

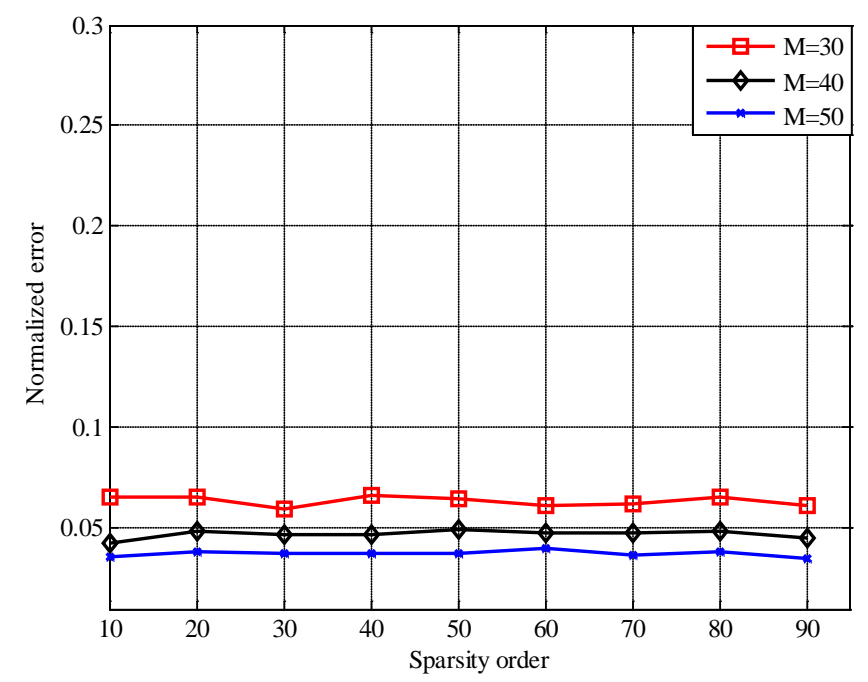

Fig. 5. The normalized error (NE) versus the sparsity of signal for different number of compressed measurements

\subsection{Simulation Results for SNR of the Compressed Signal}

To verify our theoretical analysis about $f(c)$ presented in Section 4, the theoretical and simulated PDF of $c_{i j}=\operatorname{dot}\left(\boldsymbol{\Theta}_{i}, \boldsymbol{\Theta}_{j}\right)=\left(\boldsymbol{\Theta}^{T} \boldsymbol{\Theta}\right)_{i j}, i \neq j$ are shown in Fig. 6. The theoretical curve is obtained by solving equation (16) and equation (17). It can be seen that the simulation results fit our forementioned theoretical analysis curve well. The number of compressed measurements is $M=30\left(R=30 / 512 \times R_{n q}\right)$ for Fig. 6(a), and $M=40\left(R=40 / 512 \times R_{n q}\right)$ for Fig. 6(b). It can be noticed that the variance of $c$ decreases with increasing of the number of compressed measurements. In other words, $\left(\boldsymbol{\Theta}^{T} \boldsymbol{\Theta}\right)_{i j}$ will approach 0 closely if we increase the compressed measurements. 


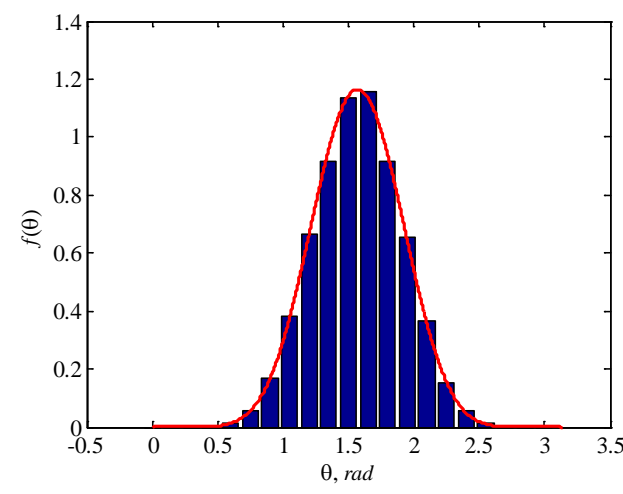

(a) $R=30 / 512 \times R_{n q}$

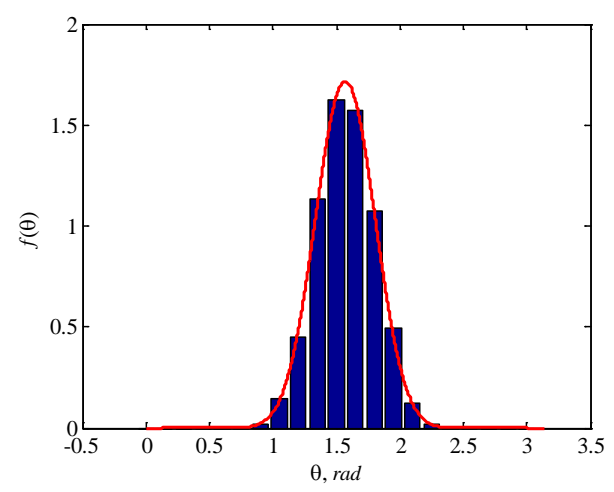

(b) $R=40 / 512 \times R_{n q}$

Fig. 6. Theoretical and simulated PDF of $c_{i j}=\operatorname{dot}\left(\boldsymbol{\Theta}_{i}, \boldsymbol{\Theta}_{j}\right)=\left(\Theta^{T} \boldsymbol{\Theta}\right)_{i j}, i \neq j$

Mathematical expectation $E\left(c^{2}\right)$ versus the number of compressed measurements is shown in Fig. 7. It can be seen that $E\left(c^{2}\right)$ varies with the number of compressed measurements inversely. If the row number of measurement matrix $\boldsymbol{\Theta}$ increases, expectation $E\left(c_{i j}^{2}\right)=E\left[\operatorname{dot}\left(\Theta_{i}, \Theta_{j}\right)\right]^{2}, i \neq j$ drops. Meanwhile, $E\left(c^{2}\right)$ will approach 0 if the number of compressed measurements becomes quite large.

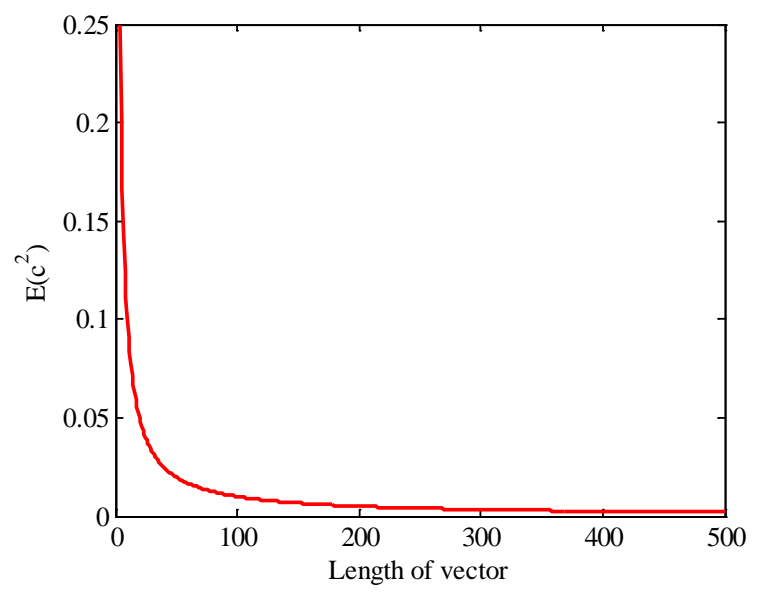

Fig. 7. Mathematical expectation $E\left(c^{2}\right)$ versus the number of compressed measurements

The SNR of the compressed signal versus the number of compressed measurements is illustrated in Fig. 8, where $K=10,20,30$ respectively, and $M$ is from 0 to 150 . The three lines denote the performance for the different sparsity of signal. It can be observed from Fig. 8 that the $S N R$ of the compressed signal varies with the number of compressed measurements directly. Since the $S N R$ of the non-compressed signal is set to $30 \mathrm{~dB}$, $S N R$ of the compressed signal is consistently lower than $30 \mathrm{~dB}$, which means that the decreasing in the number of compressed sensing is at the expense of decreasing in SNR. Meanwhile, the line with sparsity $K=10$ is consistently higher than any other line. Therefore, the $S N R$ of the compressed signal varies with the sparsity of signal inversely. 


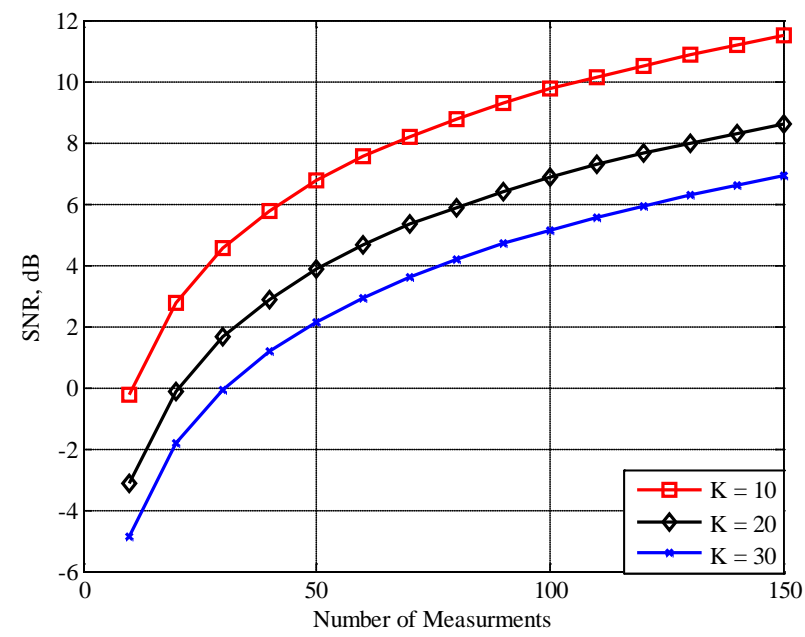

Fig. 8. SNR of the compressed signal versus the number of compressed measurements for the different sparsity of signal: $K=10,20,30$

\subsection{Simulation Results for Adaptive Adjustment in the Number of Compressed Measurements}

To evaluate the performance of the proposed method of adaptive adjustment in the number of compressed measurements, obtained measurements are applied to perform spectrum sensing. The probability of detection $P_{d}$ and probability of false-alarm $P_{f}$ serve as the evaluation criteria. We define $\boldsymbol{d}$ as a $N \times 1$ vector, which is the pratical situation of each channel. Accordingly, $\hat{\boldsymbol{d}}(N \times 1)$ is the estimated situation. The entries of $\boldsymbol{d}$ and $\hat{\boldsymbol{d}}$ are either 1(busy channel) or 0(idle channel). Then $P_{d}$ and $P_{f}$ are expressed as

$$
\begin{gathered}
P_{d}=\frac{\boldsymbol{d}^{T} \times(\boldsymbol{d}=\hat{\boldsymbol{d}})}{\mathbf{1}^{T} \times \boldsymbol{d}} \\
P_{f}=\frac{(1-\boldsymbol{d})^{T} \times(\boldsymbol{d} \neq \hat{\boldsymbol{d}})}{N-1^{T} \times \boldsymbol{d}}
\end{gathered}
$$

The $P_{d}$ and $P_{f}$ for the different sparsity of signal are illustrated in Fig. 9(a), and the number of compressed measurements versus the sparsity of signal is depicted in Fig. 9(b), where the $S N R$ of non-compressed signal is $30 \mathrm{~dB}$. From Fig. 9 (b), the adaptive method can change the number of compressed measurements with different sparsity of signal adaptively, while the other one fixes the measurements at $50\left(R=50 / 512 \times R_{n q}\right)$. According to Fig. 9 (a), when the sparsity of signal is lower, the fixed method shows better performance than the adaptive method, because the number of compressed measurements for the fixed method is larger than that of adaptive one. However, the probability of detection $P_{d}$ for fixed method experiences a dramatic fall when the sparsity of signal increases, and spectrum sensing performance of the proposed method can consistently stay on a high level in the case of possessing an acceptable computational complexity. 


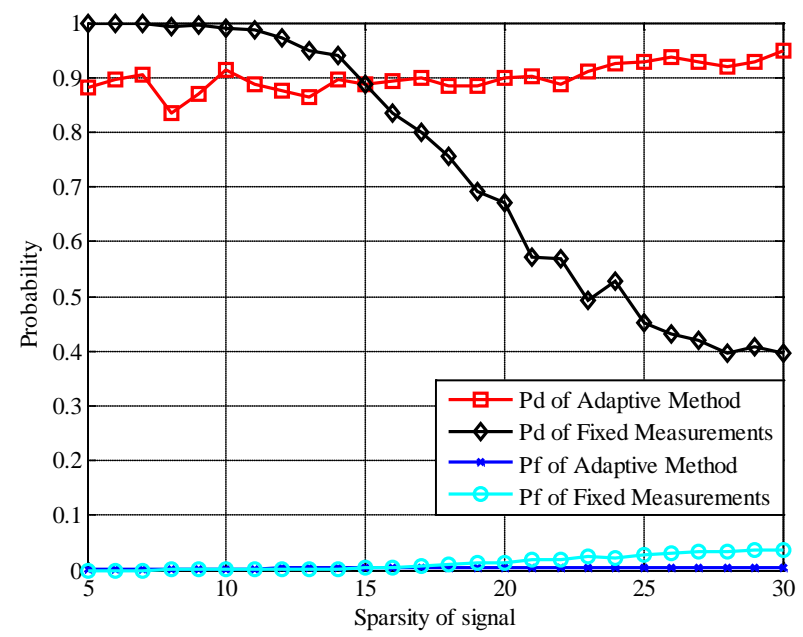

(a) The probability of detection and probability of false alarm versus sparsity

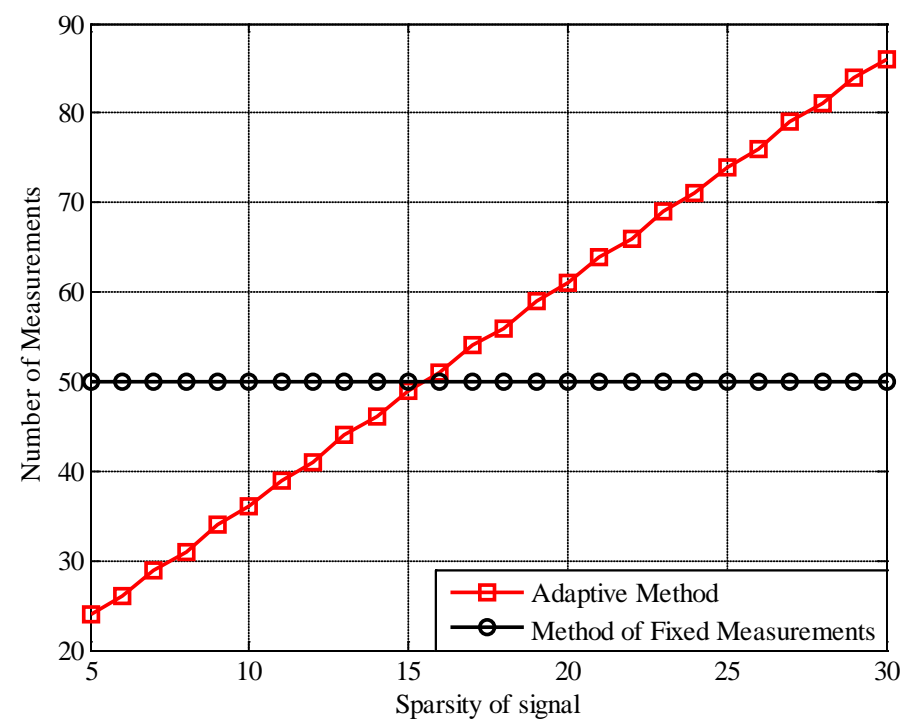

(b) The number of compressed measurements versus the sparsity of signal

Fig. 9. The performance of adaptive method and fixed method

The performance of the proposed adaptive method is compared with the case of $M=1.7 \times K \log (N / K+1)$ provided in [23] in Fig. 10, where the SNR of non-compressed signal is $30 \mathrm{~dB}$. It can be shown that the proposed method can adjust the number of compressed measurements with sparsity of signal adaptively. Moreover, the proposed method have better performance and more advantages when it is applied in spectrum sensing. 


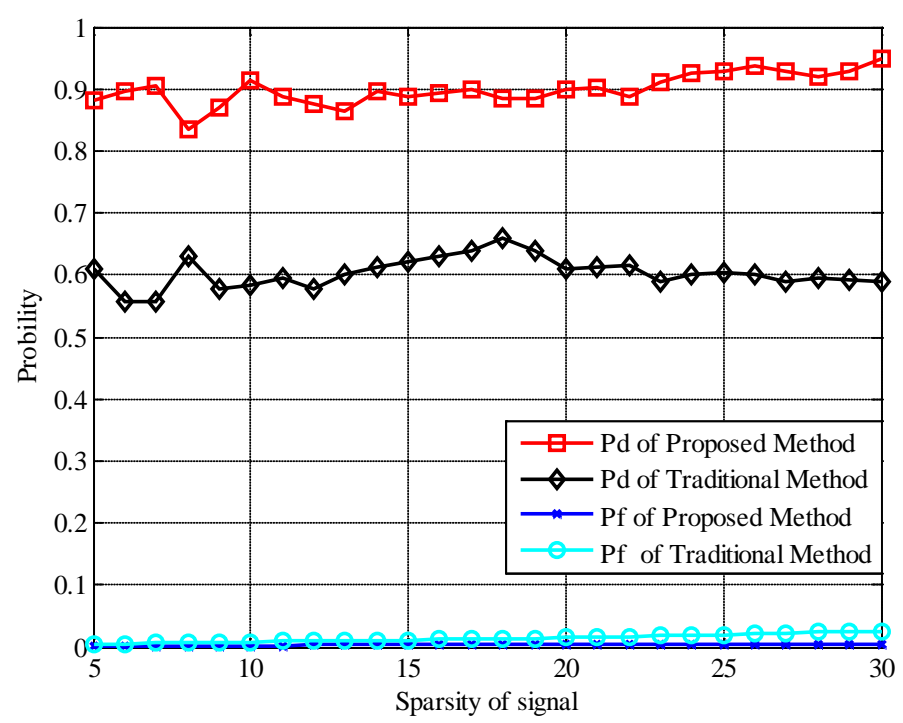

(a) Pd and Pf versus sparsity.

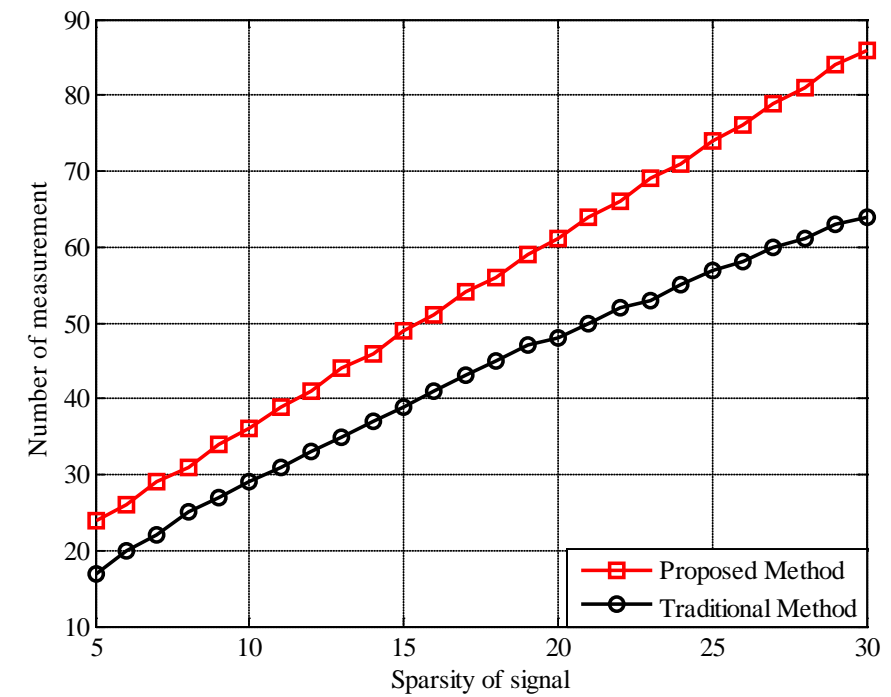

(b) The number of compressed measurements versus the sparsity of signal.

Fig. 10. The performance of the proposed adaptive method and $M=1.7 \times K \log (N / K+1)$

Our adaptive adjustment of compressed measurements is to guarantee the same $S N R$ of the compressed signal, i.e., spectrum sensing performance. If the $S N R$ of the non-compressed signal fluctuates, the proposed method can also adjust the number of compressed measurements to guarantee the spectrum sensing performance. Fig. 11 illustrates the performance of the proposed adaptive method for the different $S N R$ of the non-compressed signal, where $K=20$, the $S N R$ is from $10 \mathrm{~dB}$ to $30 \mathrm{~dB}$. 


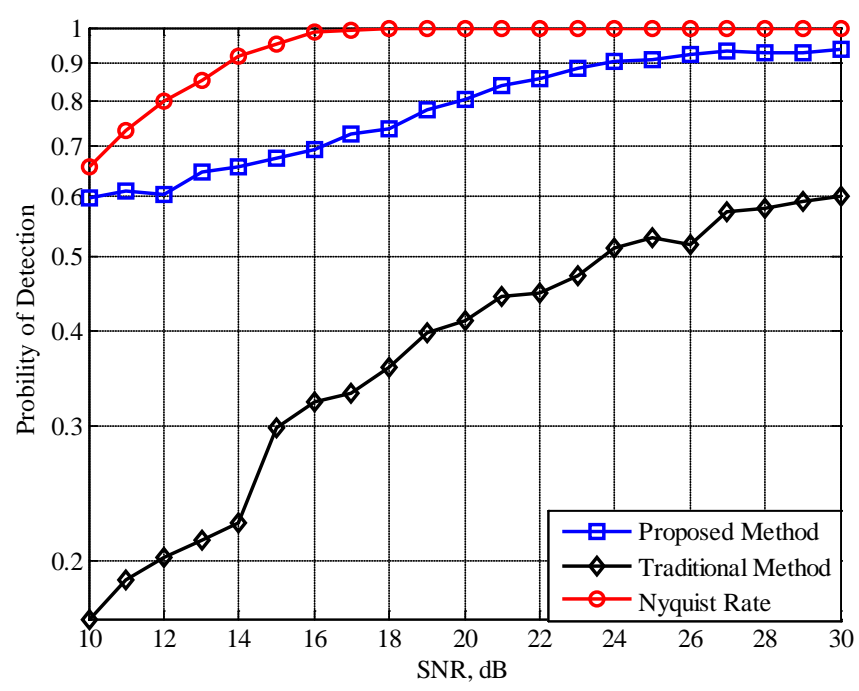

(a) The probability of detection versus SNR

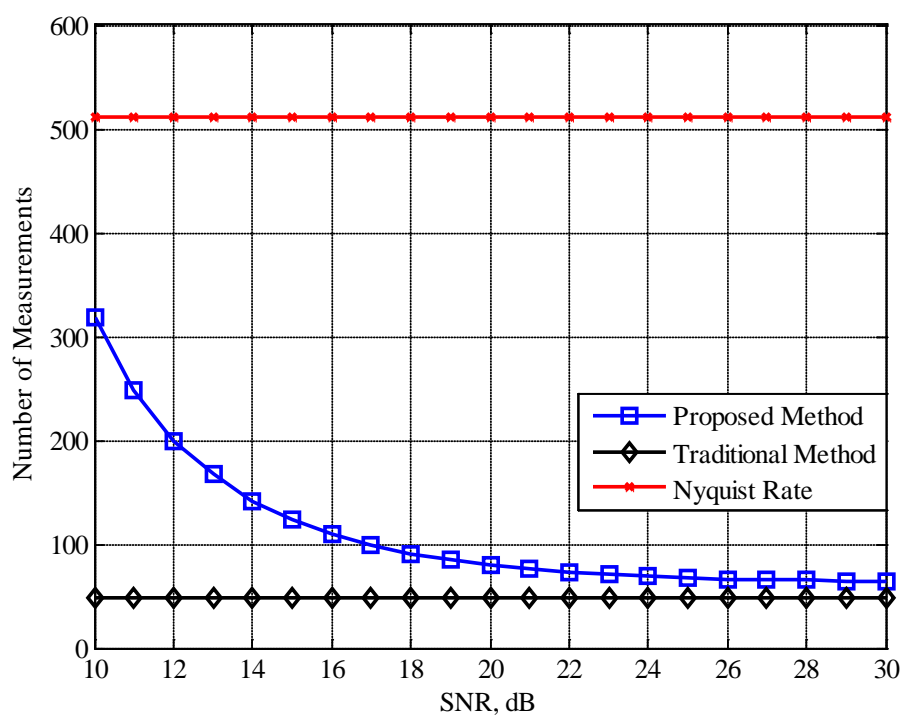

(b) The number of compressed measurements versus $S N R$

Fig. 11.The performance of the proposed adaptive method versus $S N R$ with $P_{f}$ is fixed at 0.05

From Fig. 11(a), we can see that spectrum sensing performance of the proposed method approaches that of Nyquist rate method when the SNR of the non-compressed signal increases. However, the traditional method shows degraded performance, especially in the case of low $S N R$. According to Fig. 11(b), the proposed method increases the number of compressed measurements for low SNR of the non-compressed signal, therefore possessing better performance than traditional methods. Meanwhile, the required measurements of the proposed method and traditional method are similar when the SNR of the non-compressed signal is high, which saves the computational soureces. In summary, the strategy in [32] only can be exploited in high $S N R$, while the proposed method can change the number of compressed measurements to ensure the spectrum sensing performance in low $S N R$. 


\section{Conclusion}

In this paper, a method of adaptively adjusting compressed measurements for wideband spectrum sensing was proposed in the case of unknowing the number of occupied bands (sparsity of signal in the frequency domain). Aiming at the case of unkown sparsity in the practical cognitive radio, a method of sparsity estimation was discussed, and the relationship between the amount of used data and estimation accuracy was established to evaluate the performance of estimation. Since the SNR of the compressed signal determines spectrum sensing performance, the $S N R$ of the compressed signal was derived in a closed form to analyze the factors of impacting on sensing performance. It has been observed that the SNR of the compressed signal is influenced by the SNR of the non-compressed signal, the sparsity of signal and the number of compressed measurements. Based on the previous discussion about the SNR of the compressed signal, the method of adjusting the number of compressed measurements was proposed, which possesses minimum computational complexity to ensure the required spectrum sensing performance.

\section{References}

[1] B.Wang and K.J.R.Liu, "Advances in Cognitive Radio Networks: A Survey,” IEEE Journal of Selected Topics in Signal Processing, vol.5, no.1, pp.5-23, Feb 2011. Article (CrossRef Link)

[2] C.Zou and C.Chigan, "Dynamic Spectrum Allocation Based Confidentiality for Cognitive Radio Networks,” IEEE GlobeCom 2010, pp.1-6, Dec 2010. Article(CrossRef Link)

[3] E.Axell, G.Leus, and E.G.Larsson et al, "Spectrum Sensing for Cognitive Radio:State-of-the-Art and Recent Advances,” IEEE Signal Processing Magazine, vol.29, no.3, pp.101-11, May 2012. Article(CrossRef Link)

[4] B.Farhang-Boroujeny, "Filter Bank Spectrum Sensing for Cognitive Radios," IEEE Transactions on Signal Processing, vol.56, no.5, pp.1801-1811, May 2008. Article(CrossRef Link)

[5] Y.Hur, J.Park, W.Woo, K.Lim, C.H.Lee, H.S.Kim, and J.Laskar, "A Wideband Analog Multi-resolution Spectrum Sensing Technique for Cognitive Radio Systems," IEEE Int.Symp.Circuits and Systems(ISCAS), pp.4090-4093, May 2006. Article (CrossRef Link)

[6] S.E.El-Khamy, M.S.El-Mahallawy, and E.S.Youssef, "Improved Wideband Spectrum Sensing Techniques Using Wavelet-based Edge Detection for Cognitive Radio,” 2013 International Conference on Computing, Networking and Communications (ICNC), pp.418-423, Jan 2013. Article (CrossRef Link)

[7] M.Sanna and M.Murroni, "Optimization of Non-convex Multiband Cooperative Sensing with Genetic Algorithms," IEEE Journal of Selected Topics in Signal Processing, vol.5, no.1, pp.87-96, Feb 2011. Article (CrossRef Link)

[8] K.Koufos, K.Ruttik, and R.D.Jantti, "Distributed Sensing in Multiband Cognitive Networks," IEEE Transactions on Wireless Communications, vol.10, no.5, pp.1667-1677, May 2011. Article (CrossRef Link)

[9] R.Daniel and L.Geert, "Wideband Spectrum Sensing from Compressed Measurements Using Spectral Prior Information," IEEE Transactions on Signal Processing, vol.61, no.24, pp. 6232-6246, Dec 2013. Article (CrossRef Link)

[10] Y.Wang, C.Guo, X.Sun, and C.Feng, "Time-efficient Wideband Spectrum Sensing Based on Compressive Sampling,” 2015 IEEE 81st Vehicular Technology Conference (VTC Spring), pp.1-5, May 2015. Article (CrossRef Link)

[11] D.L.Donoho, "Compressed Sensing," IEEE Transactions on Information Theory, vol.52, no.4, pp.1289-1306, Apr 2006. Article (CrossRef Link)

[12] M.F.Duarte and Y.C.Eldar, "Structured Compressed Sensing: from Theory to Applications," IEEE Transactions on Signal Processing, vol.59, no.9, pp.4053-4085, Sep 2011. Article (CrossRef Link) 
[13] E.J.Candes and T.Tao, "Near-optimal Signal Recovery from Random Projections: Universal Encoding Strategies,” IEEE Transactions on Information Theory, vol.52, no.12, pp. 5406-5425, Dec 2006. Article (CrossRef Link)

[14] Z.Tian and G.B.Giannakis, "Compressed Sensing for Wideband Cognitive Radios," IEEE International Conference on Acoustics, Speech and Signal Processing (ICASSP), pp.1357-1360, Apr 2007. Article (CrossRef Link)

[15] T.Zhi, Y.Tafesse and B.M.Sadle, "Cyclic Feature Detection With Sub-Nyquist Sampling for Wideband Spectrum Sensing,” IEEE Journal of Selected Topics in Signal Processing, vol.6, no.1, pp.58-69, Feb 2012. Article (CrossRef Link)

[16] Z.Zhang, Z.Han, H.Li, D.Yang, and C.Pei, “ Belief Propagation Based Cooperative Compressive Spectrum Sensing in Wideband Cognitive Radio Networks," IEEE Transactions on Wireless communications, vol.10, no.9, pp.3020-3031, Sep 2011. Article (CrossRef Link)

[17] M.A.T.Fiqueiredo, R.D.Nowak, and S.J.Wright, "Gradient Projection for Sparse Reconstruction: Application to Compressed Sensing and Other Inverse Problems," IEEE Journal of Selected Topics in Signal Processing, vol.1, no.4, pp.586-597, Dec 2007. Article (CrossRef Link)

[18] J.A.Tropp and A.C.Gilbert, "Signal Recovery from Random Measurements via Orthogonal Matching Pursuit,” IEEE Transactions on Information Theory, vol.53, no.12, pp.4655-4666, Dec 2007. Article (CrossRef Link)

[19] D.Needell and J.A.Tropp, "CoSaMP: Iterative Signal Recovery from Incomplete and Inaccurate Samples," Applied and Computational Harmonic Analysis, vol.26, no.3, pp.301-321, May 2009. Article (CrossRef Link)

[20] D.L.Donoho, Y.Tsaig, Droril, and J.L.Starck, "Sparse Solution of Underdetermined Linear Equations by Stagewise Orthogonal Matching Pursuit,” IEEE Transactions on Information Theory, vol.58, no.2, pp.1094-1121, Feb 2012. Article (CrossRef Link)

[21] C.Fu, X.Ji, and Q.Dai, “Adaptive Compressed Sensing Recovery Utilizing the Property of Signal's Autocorrelations,” IEEE Transactions on Image Processing, vol.21, no.5, pp.2369-2378, May 2012. Article (CrossRef Link)

[22] J.Haupt, R.Nowak, and R.Castro, “Adaptive Sensing for Sparse Signal Recovery,” in Proc. of IEEE 13th Digital Signal Processing Workshop and 5th IEEE Signal Processing Education Workshop, pp.702-707, Jan 2009.Article (CrossRef Link)

[23] M.A.Iwen and A.H.Tewfik, “Adaptive Compressed Sensing for Sparse Signals in Noise,” in Proc. of IEEE 2011 Conference Record of the Forty Fifth Asilomar Conference on Signals, Systems and Computers, pp.1240-1244, Nov 2011. Article (CrossRef Link)

[24] M.L.Malloy and R.D.Nowak, "Near-optimal Adaptive Compressed Sensing," IEEE Transactions on Information Theory, vol.60, no.7, pp.4001-4012, July 2014. Article (CrossRef Link)

[25] J.Zhang, D.Zhu, and G.Zhang, “Adaptive Compressed Sensing Radar Oriented toward Cognitive Detection in Dynamic Sparse Target Scene,” IEEE Transactions on Signal Processing, vol.60, no.4, pp.1718-1729, Apr 2012. Article (CrossRef Link)

[26] Z.Liu, J.Liu, and Z.Qiu, “A New Adaptive Compressed Sensing Algorithm for Wireless Sensor Networks,” 2010 IEEE 10th International Conference on Signal Processing (ICSP), pp.2452-2455, Oct 2010. Article (CrossRef Link)

[27] J.A.Tropp, J.N.Laska, and M.F.Duarte et al, "Beyond Nyquist: Efficient Sampling of Sparse Bandlimited Signals,” IEEE Transactions on Information Theory, vol.56, no.1, pp.520-544, Jan 2010. Article (CrossRef Link)

[28] W.Tlan, G.Rui, and J.Kanf, "Divide and Conquer Method for Sparsity Estimation within Compressed Sensing Framework,” Electronics Letters, vol.50, no.9, pp.677-678, Apr 2014. Article (CrossRef Link)

[29] S.K.Sharma, S.Chatzinotas, and B.Ottersten, "Compressive Sparsity Order Estimation for Wideband Cognitive Radio Receiver,” IEEE Transactions on Signal Processing, vol.62, no.19, pp.4984-499, Oct 2014. Article (CrossRef Link)

[30] Y.Wang, Z.Tian, and C.Feng, "Sparsity Order Estimation and Its Application in Compressive Spectrum Sensing for Cognitive Radios,” IEEE Transactions on Wireless Communications, vol.11, no.6, pp.2116-2125, Jun 2012. Article (CrossRef Link) 
[31] Y.Wang, Z.Tian, and C.Feng, “A Two-step Compressed Spectrum Sensing Scheme for Wideband Cognitive Radios,” in Proc. of 2010 IEEE Global Telecommunications Conference, pp.1-5, Dec 2010. Article (CrossRef Link)

[32] X.Zhu, J.Wang, and L.Dai et al, "Sparsity-aware Adaptive Channel Estimation Based on SNR Detection,” IEEE Transactions on Broadcasting, vol.61, no.1, pp.119-126, Mar 2015. Article (CrossRef Link)

[33] T.Cai, J.Fan, and T.Jiang, "Distributions of Angles in Random Packing on Spheres," The Journal of Machine Learning Research, vol.14, no.1, pp.1837-1864, Jun 2013. Article (CrossRef Link)

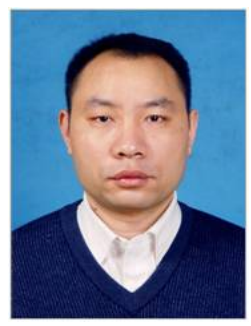

Yulong Gao is an associate professor in School of Electronics and Information Engineering, Harbin Institute of Technology, Harbin, P.R.China. He received the doctor degree in School of Electronics and Information Engineering from Harbin Institute of Technology in 2007. From 2008 to 2010, he was a postdoctoral researcher in Harbin Institute of Technology. From 2012 to 2013, Dr. Yulong Gao worked in Department of Electrical \& Computer Engineering, University of Toronto as a visiting scholar. His main research fields are communication signal processing and compressed sensing.

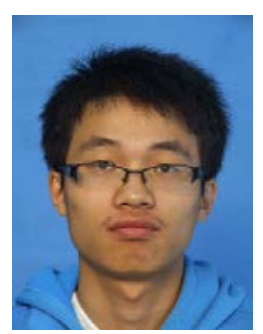

Wei Zhang is a doctor student in Department of Electronic Engineering, City University of Hong Kong. He received the Bachelor degree and Master degree in School of Electronics and Information Engineering from Harbin Institute of Technology, Harbin, P.R.China in 2013 and 2015, respectively. His research interests include spectrum detection of cognitive radio and compressed sensing.

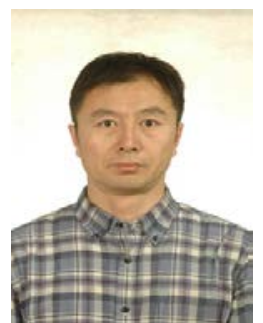

Yongkui Ma is an associate professor in School of Electronics and Information Engineering, Harbin Institute of Technology. He received the doctor degree in School of Electronics and Information Engineering from Harbin Institute of Technology in 2004. His current research interests include statistical signal processing and compressed sensing. 